\title{
GATEAUX DIFFERENTIABLE POINTS WITH SPECIAL REPRESENTATION
}

\author{
SEUNG JAE OH
}

\begin{abstract}
If $\left(x_{n}\right)$ is a bounded sequence in a Banach space, is there an element $x=\sum_{n=1}^{\infty} a_{n} x_{n}$ such that $\sum_{n=1}^{\infty}\left\|a_{n} x_{n}\right\|<\infty$ and the directional derivative of the norm at $x, D\left(x, x_{n}\right)$, exists for every $n$ ? In fact, there are such $x$ 's dense in the closed span of $\left\{x_{n}\right\}$. An application of this fact is made to a proof of Rybakov's theorem on vector measures.
\end{abstract}

A real-valued function $f$ defined on a linear topological space $X$ is said to be Gateaux differentiable at $x \in X$ if, for every $y \in X$,

$$
D f(x ; y)=\lim _{t \rightarrow 0} \frac{f(x+t y)-f(x)}{t}
$$

exists and converges to $x^{*}(y)$ for a unique $x^{*} \in X^{*}$, where $X^{*}$ denotes the linear space of all continuous linear functionals on $X$. If $D f(x ; y)$ exists for a particular direction $y \in X$, then we call this limit the Gateaux derivative of $f$ at $x$ in the direction of $y$. The notation for the directional derivative of the norm is

$$
D(x ; y)=\lim _{t \rightarrow 0} \frac{\|x+t y\|-\|x\|}{t} .
$$

A real-valued function $f$ on a subset $A$ of a linear space $X$ is said to be subdifferentiable at $x \in A$ if there exists $x^{*} \in X^{*}$ such that

$$
x^{*}(y-x) \leqslant f(y)-f(x) \text { for all } y \text { in } A \text {. }
$$

We say that $x^{*}$ is a subgradient of $f$ at $x$. We denote by $\partial f(x)$ the set of all subgradients of $f$ at $x$ and call this set the subdifferential of $f$ at $x$.

THEOREM 1. Let $f$ be a continuous convex function defined on a Banach space $X$, and $\left(x_{n}\right)$ a bounded sequence in $X$; then there is an element $x=\sum_{n=1}^{\infty} a_{n} x_{n}$ such that $\sum_{n=1}^{\infty}\left\|a_{n} x_{n}\right\|<\infty$ and $D f\left(x ; x_{n}\right)$ exists for every $n$. Further, such $x$ 's are dense in the closed span of $x_{n}$ 's.

Proof. Suppose $\left(x_{n}\right)$ is a bounded sequence in $X$ and $f$ is a continuous convex function on $X$. Define $T: l_{1} \rightarrow X$ by

$$
T(a)=\sum_{n=1}^{\infty} a_{n} x_{n} \quad \text { where } a=\left(a_{n}\right) \in l_{1} .
$$

Received by the editors March 2, 1984 and, in revised form, June 5, 1984.

1980 Mathematics Subject Classification. Primary 46A55; Secondary 46A32, 52A07.

Key words and phrases. Continuous convex function, Gateaux differentiable, countably additive measure. 
The norm of $T$ is the surpemum of the norms of $x_{n}$,s, so $T$ is a continuous linear operator and $f \circ T$ is a continuous convex function on the separable space $l_{1}$. By Mazur's theorem [4] there is a dense $G_{\delta}$ subset $G \cong l_{1}$ at each point of which $f \circ T$ is Gateaux differentiable. By the continuity of $T$ the set $D=T(G)$ is a dense subset of $T\left(l_{1}\right)$, which is, in turn, dense in the closed span $S$ of $\left\{x_{n}\right\}$. Thus, $D$ is dense in the latter. If $x_{1}^{*}$ and $x_{2}^{*}$ are elements of the subdifferential $(\partial f)(T a)$ for some $a \in G$, then

$$
x_{i}^{*}(x-T a) \leqq f(x)-f(T a) \text { for all } x \in X, i=1,2 .
$$

In particular,

$$
x_{i}^{*}(T b-T a) \leqq f(T b)-f(T a) \text { for all } b \in l_{1}, i=1,2,
$$

which means $x_{1}^{*} \circ T$ and $x_{2}^{*} \circ T$ are in $\partial(f \circ T)(a)$. But $f \circ T$ is Gateaux differentiable on $G$, and, hence, $x_{1}^{*} \circ T=x_{2}^{*} \circ T$ on $l_{1}$. This implies that $x_{1}^{*}=x_{2}^{*}$ on $T\left(l_{1}\right)$ and also on $S$; that is, $f$ restricted to $S$ is Gateaux differentiable at each point of the dense set $D$ in $S$ since $\partial f(T a)$ is a singleton set [3, p. 122]. $D$ has the form $\sum_{n=1}^{\infty} a_{n} x_{n}$ with $\sum_{n=1}^{\infty}\left\|a_{n} x_{n}\right\|<\infty$, so the proof is complete.

This theorem applies to spaces of measures. Let $\Sigma$ be a $\sigma$-field of subsets of the point set $\Omega$, and let $X$ be a Banach space. The space $\operatorname{ca}(\Sigma, X)$ consists of all countably additive $X$-valued measures on $\Sigma$ normed by the semivariation. The following is an obvious corollary.

COROLlaRY. If $\left(\mu_{n}\right)$ is a bounded sequence in $\mathrm{ca}(\Sigma, X)$, then there is an element $\mu=\sum_{n=1}^{\infty} a_{n} \mu_{n}$ such that $\sum_{n=1}^{\infty}\left\|a_{n} \mu_{n}\right\|<\infty$ and $D\left(\mu, \mu_{n}\right)$ exists for every $n$.

Let $F \in \operatorname{ca}(\Sigma, X)$, and let $\mu$ be a finite nonnegative real-valued measure on $\Sigma$. $F$ is called $\mu$-continuous $(F \ll \mu)$ if $\lim _{\mu(E) \rightarrow 0} F(E)=0$. We now state Rybakov's theorem. (|| stands for the variation norm.)

TheOREM 2 (Rybakov [5]). If $F \in \operatorname{ca}(\Sigma, X)$, then there is an element $x^{*} \in X^{*}$ such that $F \ll\left|x^{*} F\right|$.

In order to prove the theorem we need to use the following Lemma. A proof may be found in [2, pp. 11-13]. We also use the key fact [1] that if $\lambda, \mu \in \operatorname{ca}(\Sigma, R)$ and $D(\lambda, \mu)$ exists, then $\mu \ll \lambda$.

LEMma. If $F \in \mathrm{ca}(\Sigma, X)$, then there exists a nonnegative real-valued countably additive measure $\mu$ on $\Sigma$ such that $F \ll \mu$. Moreover, $\mu$ can be chosen so that $\mu=\sum_{n=1}^{\infty} \beta_{n}\left|x_{n}^{*} F\right|$ for some $\left(x_{n}^{*}\right)$, with $\left\|x_{n}^{*}\right\|=1$ for all $n$, where the $\beta_{n}$ 's can be selected to be nonnegative and satisfy $\sum_{n=1}^{\infty} \beta_{n}=1$.

Proof of Theorem 2. By using the Lemma let $F \ll \sum_{n=1}^{\infty} \beta_{n}\left|x_{n}^{*} F\right|$, where $\left(x_{n}^{*} F\right)$ is a bounded sequence in $\operatorname{ca}(\Sigma, R)$. Hence, by the Corollary, there is an element $\mu$ in $\operatorname{ca}(\Sigma, R)$ such that $\mu=\sum_{n=1}^{\infty} a_{n}\left(x_{n}^{*} F\right)$ and $D\left(\mu, x_{n}^{*} F\right)$ exists for every $n$. If we set $x^{*}=\sum_{n=1}^{\infty} a_{n} x_{n}^{*}$, then $x^{*} F=\sum_{n=1}^{\infty} a_{n} x_{n}^{*} F$. Now since $D\left(x^{*} F, x_{n}^{*} F\right)$ exists for all $n$, we have $\left|x_{n}^{*} F\right| \ll\left|x^{*} F\right|$ for all $n[1]$. Therefore,

$$
F \ll \sum_{n=1}^{\infty} \beta_{n}\left|x_{n}^{*} F\right| \ll\left|x^{*} F\right| .
$$




\section{REFERENCES}

1. R. G. Bilyeu and P. W. Lewis, Orthogonality and Hewitt-Yosida theorem in spaces of measures, Rocky Mountain J. Math. 7 (1977), 629-638.

2. J. Diestel and J. J. Uhl, Jr., Vector measures, Amer. Math. Soc., Providence, R. I., 1970.

3. J. R. Giles, Convex analysis with application in differentiation of convex functions, Pitman, Boston, Mass., 1982.

4. S. Mazur, Über konvexe mengen in linearen normierte raumen, Studia Math. 4 (1933), 70-84.

5. V. Rybakov, Theorem of Bartle, Dunford, and Schwartz on vector-valued measures, Mat. Zametki 7 (1970), 247-254.

Department of Mathematics, Hannam University, Taejeon, Choongnam, Korea 